\title{
Posibles complicaciones del SARS-COV-2 sobre las funciones neurocognitivas.
}

\author{
Possible complications of SARS-COV-2 on neurocognitive functions.
}

\author{
Sheila Castro-Suarez ${ }^{1,2}$, Nilton Custodio ${ }^{2,3,4}$
}

Desde los primeros reportes de la COVID-19 se reconoce a la neumonia intersticial como manifestación de la infección por el coronavirus del síndrome respiratorio agudo severo 2 (SARS-CoV-2) (1). Sin embargo, en la actualidad es ampliamente conocido que el SARS-CoV-2 se une al receptor de la enzima convertidora de angiotensina 2 para ingresar a las células humanas, como las neuronas y las células gliales lo que explicaría la amplia gama de manifestaciones neurológicas que se presentan a lo largo del proceso de la enfermedad (2).

Es probable que varios mecanismos de la COVID-19 repercutan en la cognición (3). Se ha propuesto mecanismos directos e indirectos que pueden explicar el daño en el sistema nervioso central; el efecto neurotropico del SARS$\mathrm{CoV}-2$, las complicaciones trombóticas, inflamatorias y la desregulación de la presión arterial propagan el daño neurológico reportado y podrían conducir a un deterioro del funcionamiento cognitivo (4). A estos mecanismos se suma las consecuencias de la hipoxia derivadas de los casos de COVID-19 gravemente afectados; el daño isquémico que puede generar en la sustancia blanca cerebral es de importancia crítica para la función cognitiva $(3,5)$. Las estructuras cerebrales límbicas y asociadas como el hipocampo y los ganglios basales contienen más enzimas que están involucradas en las respuestas inflamatorias que otras áreas. Por lo tanto, existe un mayor riesgo de desarrollar déficits en procesos neurocognitivos como la memoria y la atención (6). Por otro lado, se conjetura que el SARS-CoV-2 puede acelerar el proceso neurodegenerativo de las demencias. Cada vez hay más pruebas de que la hipoperfusión cerebral acelera la acumulación de amiloide- $\beta$ y está relacionada con la patología de tau y TDP43 (3). La COVID-19 y la enfermedad de Alzheimer comparten vínculos comunes con respecto a los receptores de la ECA2, los marcadores proinflamatorios y el alelo APOE4 (7). Por lo que las habilidades cognitivas y la funcionalidad de las personas puede verse comprometida a largo plazo.

Las manifestaciones neurológicas de la COVID-19 han sido reportadas hasta en un 36.4\%; la población que presenta estas manifestaciones, es aquella con infección grave y con riesgo cardiovascular subyacente como la hipertensión arterial (8). La presencia de síntomas neurológicos durante la infección, como el dolor de cabeza, anosmia y disgeusia, pueden ser factores de riesgo para el deterioro cognitivo relacionado con la atención, la memoria y la función ejecutiva (6). Estudios recientes vienen investigando los síntomas a largo plazo en pacientes que se infectaron con el SARS-CoV-2, y dentro de sus resultados muestran a los trastornos de la atención (27\%); y el compromiso de memoria en un 16\% (9). De igual forma, un estudio que incluyó población peruana mayor de 55 años reportó que los problemas de memoria influyen en la salud de las personas que fueron infectadas por la COVID-19 (10). Se debería considerar la identificación y evaluación de estos pacientes a largo plazo para prevenir un mayor deterioro y compromiso en la ejecución de las actividades cotidianas, incluyendo dificultades laborales, con gran repercusión funcional y emocional no sólo para el propio paciente sino en su entorno familiar (6).

Centro de Investigación Básica en Demencia y Enfermedades desmielinizantes del Sistema Nervioso, Instituto Nacional de Ciencias Neurológicas. Lima, Perú.

2 Departamento de Investigación y docencia, Instituto Peruano de Neurociencias. Lima, Perú.

3 Servicio de Neurología, Instituto Peruano de Neurociencias. Lima, Perú.

4 Unidad de diagnóstico de deterioro cognitivo y prevención de demencia, Instituto Peruano de Neurociencias. Lima, Perú. 
La evidencia actual sugiere que pacientes infectados con el SARS-CoV-2 y logran sobrevivir a la COVID-19 tienen un alto riesgo de desarrollar enfermedades neurológicas, en particular el compromiso cognitivo; ya sea como resultado de la aceleración o de la inducción de mecanismos neurodegenerativos de novo. Se necesitan más estudios para investigar las posibles correlaciones entre las infecciones agudas y subagudas por COVID-19 y las secuelas neurológicas a largo plazo.

\section{REFERENCIAS BIBLIOGRÁFICAS}

1. Mohanty SK, Satapathy A, Naidu MM, Mukhopadhyay S, Sharma S, Barton LM, et al. Severe acute respiratory syndrome coronavirus-2 (SARSCoV-2) and coronavirus disease 19 (COVID-19) anatomic pathology perspective on current knowledge. Diagn Pathol. 2020;15:103. doi: 10.1186/ s13000-020-01017-8

2. Zhou Z, Kang H, Li S, Zhao X. Understanding the neurotropic characteristics of SARS-CoV-2: from neurological manifestations of COVID-19 to potential neurotropic mechanisms. JNeurol.2020;267(8):217984. doi: 10.1007/s00415-020-09929-7

3. Miners S, Kehoe PG, Love S. Cognitive impact of COVID-19: looking beyond the short term. Alzheimers Res Ther. 2020;12(1):170. doi: 10.1186/ s13195-020-00744-w

4. Wenting A, Gruters A, van Os Y, Verstraeten S, Valentijn S, Ponds R, et al. COVID-19 Neurological Manifestations and Underlying Mechanisms: A Scoping Review. Front Psychiatry. 2020;11:860. doi: 10.3389/fpsyt.2020.00860

5. Needham EJ, Chou SH-Y, Coles AJ, Menon DK. Neurological Implications of COVID-19 Infections. Neurocrit Care. 2020;32(3):667-71. doi: 10.1007/ s12028-020-00978-4.
6. Almeria M, Cejudo JC, Sotoca J, Deus J, Krupinski J. Cognitive profile following COVID-19 infection: Clinical predictors leading to neuropsychological impairment. Brain Behav Immun Health. 2020;9:100163. doi: 10.1016/j.bbih.2020.100163

7. Rahman MA, Islam $\mathrm{K}$, Rahman S, Alamin M. Neurobiochemical Cross-talk Between COVID-19 and Alzheimer's Disease. Mol Neurobiol. 2021;58(3):1017-23. doi: 10.1007/s12035-02002177-w

8. Mao L, Jin $\mathrm{H}$, Wang $\mathrm{M}, \mathrm{Hu} \mathrm{Y}$, Chen $\mathrm{S}, \mathrm{He}$ Q, et al. Neurologic Manifestations of Hospitalized Patients With Coronavirus Disease 2019 in Wuhan, China. JAMA Neurol. 2020;77(6):683-90. doi: 10.1001/ jamaneurol.2020.1127

9. Lopez-Leon S, Wegman-Ostrosky T, Perelman C, Sepulveda R, Rebolledo PA, Cuapio A, et al. More than 50 Long-term effects of COVID-19: a systematic review and meta-analysis. medRxiv. 2021; 2021.01.27.21250617. doi: 10.1101/2021.01.27.212 50617

10. Babulal GM, Torres VL, Acosta D, Agüero C, Aguilar-Navarro S, Amariglio R, et al. The impact of COVID-19 on the well-being and cognition of older adults living in the United States and Latin America. EClinicalMedicine. 2021;35:100848. doi: 10.1016/j.eclinm.2021.100848 